A Proposed System for the Development of an Online Learning Environment for the Initial Action of Clinical Nurses at Times of Disaster Miyuki Horiuchi ${ }^{1}$, Ryuichi Matsuba ${ }^{2}$, Makoto Miyazaki ${ }^{3}$

1. Nursing, Kio University, Kita_katsuragi, Nara/Japan

2. Kumamoto University, Kumamoto/Japan

3. Kio University, Kita_katsuragi, Nara/Japan

Study/Objective: The purpose of this poster presentation is to propose a system to support the education of nurses working in medical facilities, and to raise the capacity of disaster-resistance among regional medical institutions, using ICT (Information and Communication Technology).

Background: Sharing reviews of daily nursing among individual nurses has played an important role in the development of nursing science. How to accumulate the knowledge and skills of experienced professionals is a big challenge for those working in the fields of disaster medicine/nursing. Therefore, we conduct a project in the followings steps.

Methods: Development of nursing competencies required for emergency response, focused on disaster "initial movement".

Construction of an online learning environment using open source (Mahara), for accumulation and sharing of knowledge.

Providing online learning materials with an adequate selfevaluation method, such as portfolio or rubrics based on the competencies acquired in initial disaster nursing.

Sharing information regarding practical implementation of appropriate initial disaster nursing activities via the internet.

Results: We expect to obtain the following outcomes through the project:

Development of nursing competencies results in the standardization of disaster nursing education.

Online learning materials based on experiences of accomplished nurses, will become available for simulation learning about disaster "initial movement" in many hospitals.

Utilization of mobile devices like smart-phones, tablet terminals, and PCs can give equal learning opportunities, regardless of time and place, contributing to the improvement of knowledge and skills of nurses.

Using the Rubric analysis, it is expected that reachability will be visually indicated, and the learning plan will be easy to formulate. Conclusion: Development of a systematic educational program is required for nurses working in hospitals, to raise knowledge and skill levels on disaster medical care.

Prehosp Disaster Med 2017;32(Suppl. 1):s148

doi:10.1017/S1049023X17004071

A Qualitative Case Study to Explore how Nursing Educators Address Disaster Knowledge and Competencies in Nursing Schools in British Columbia

Wendy Mckenzie

Education, University of Calgary, Calgary Alberta/AB/Canada

Study/Objective: The purpose of this study is to explore and describe disaster-nursing curricula, particularly the development and inclusion of disaster-nursing knowledge/competencies within Schools Of Nursing (SON) in British Columbia.
Background: The province of British Columbia in Canada is experiencing an increase in both natural and human-made disasters, as evidenced by recent forest fires, mudslides, and oil spills. Nurses are known to be one of the largest groups of health care workers and are often challenged by a trifecta of roles, that of being victims themselves, first responders, as well as having acute/community employment roles. Meeting the challenges of these multiple roles can be positively influenced through disaster education; however, it is difficult to know what or how to provide this education without first knowing what nurses are currently being taught in Schools of Nursing. This qualitative case study will explore, through the lens of nursing educators, how disaster knowledge is addressed in nursing schools in British Columbia.

Methods: A qualitative case study research approach informed by Stakeholders was used to address the purpose of my research. Ten interviews were conducted with nurse educators from a variety of geographical locations across the province of British Columbia. Following ethics certifications, interview data were collected during face to face sessions, or by electronic means for this research using a semi-structured interview method. Data were managed using NVIVO for coding and thematic analysis. Results: Preliminary results are indicating that nurse educators are not teaching disaster knowledge within their programs due to inadequate time in the curriculum, not enough interest, and the educators do not feel they have enough knowledge themselves to adequately teach this knowledge. Final results for this study will be available at the WADEM conference. Conclusion: Final conclusions will be presented at the Toronto conference.

Prehosp Disaster Med 2017;32(Suppl. 1):s148

doi:10.1017/S1049023X17004083

\section{Knowledge of Cardiology Nurses, Regarding Care of Patients with Permanent Pacemakers and Implantable Cardioverter Defibrillators in Karachi Pakistan Faisal Ameen}

School Of Nursing, Aga Khan University School of Nursing and Midwifery, karachi/Pakistan

Study/Objective: The primary research question of the study was: What is the level of knowledge among cardiology nurses regarding the care of patients with permanent pacemakers and ICDs at two tertiary care hospitals in Karachi, Pakistan?

Background: It is recognized increasingly that patients with implanted PPMs or ICDs are a real challenge, especially when they require long-term care. Adequate knowledge of nurses about the care of PPMs and ICDs improves accuracy in measurement of parameters, assists in setting realistic goals and making clinically wise decisions, and improves their overall performance.

Methods: A descriptive, cross-sectional study design was selected, and a total 139 study participants were enrolled in this study from the Aga Khan University Hospital and the Tabba Heart Institute, Karachi, Pakistan. The data were collected through a structured questionnaire to assess nurses' knowledge regarding care of patients with PPMs and ICDs.

Results: The findings of the current study revealed that $26.6 \%$ of the study participants had a high knowledge regarding the 
care of patients with PPMs and ICDs, 64\% of the study participants had a moderate level of knowledge, whereas $9.4 \%$ of the study participants' knowledge was poor. The current study also found a significant difference between novice and expert nurses' knowledge; moreover, a significant difference was also found between the mean levels of knowledge score of nurses having different levels of qualification. Thus, the study also identified a significant difference between the specific study questions; however, a true comparison could not be assured, as dip card nurses were 19 in number.

Conclusion: The majority of the nurses were found to have a moderate level of knowledge regarding the care of patients with PPMs and ICDs. Furthermore, the finding of the study has created a platform for nursing leaders to start a comprehensive training program in order to improve the care quality of the patients. Prehosp Disaster Med 2017;32(Suppl. 1):s148-s149

doi:10.1017/S1049023X17004095

Stress Among the Nurses: Working in an Emergency Unit of a Tertiary Care Teaching Hospital of Eastern Nepal Ram S. Mebta

Medical-surgical Nursing, B. P. Koirala Institute of Health Sciences, Dharan/Nepal

Study/Objective: To assess the stress among the nurses working in Emergency unit of BPKIHS, and to find out the association between stress and selected demographic variables.

Background: Nurses who work in highly stressful situations are constantly under pressure, and are vulnerable to a variety of symptoms in reaction to the stress.

Methods: A cross-sectional descriptive design was used for the study. The Total Enumerative Sampling technique was used. The sample consisted of all the staffs (25) working in the critical care units. A semi-structured questionnaire was used for data collection. Verbal permission was obtained from each respondent maintaining confidentiality and anonymity. The data were collected from the nurses July 7-14, 2016. The total score of the stress components in the tool, to determine the stress, was 82 . The cut off value was taken as $50 \%$ (41), to categorize the presence or absence of stress among the respondents.

Results: Among the total 25 respondents enrolled in the study, the majority (68\%) were below 20 years of age. The majority of them (84\%) were married. Maximum respondents (72\%) worked up to 48 hours/week, less than one-half (28\%) worked more than 48 hours/week. The study showed that $12 \%$ of the respondents had stress working in critical care units, whereas $88 \%$ had no stress. Among the socio-demographic variables, the marital status of the nurses had association with stress $(\mathrm{P}=.011)$. Considering the job related variables, the over-time duty performed by the nurses per week had association with stress statistically $(\mathrm{P}=.04)$. Conclusion: This study shows that very few nurses (12\%) were experiencing stress while working in the Emergency Unit of a tertiary care teaching hospital in eastern Nepal. Apart from their marital status, no other socio-demographic variables were statistically associated to the stress experience.

Prehosp Disaster Med 2017;32(Suppl. 1):s149

doi:10.1017/S1049023X17004101
Knowledge, Attitude and Practice Study of Oxygen Therapy among Emergency Department Nurses in Addis Ababa, Ethiopia

Girma Lema ${ }^{1}$, Asefw W/Tsadik ${ }^{2}$,Lemlem Beza ${ }^{3}$

1. Yekatite 12 hospital medical college, Adis Ababa/Ethiopia

2. Addis Ababa University, Adiss Ababa/Ethiopia

3. Emergency Medicine, Addis Ababa University, Addis Ababa/ Ethiopia

Study/Objective: We aimed to identify factors impacting the appropriate use of oxygen by nurses within this setting, with a goal to improve delivery of this therapy.

Background: Oxygen $\left(\mathrm{O}_{2}\right)$ should be considered a drug as it is prescribed to prevent or treat hypoxemia. The concentration of oxygen prescribed aims to bring oxygen saturation $\left(\mathrm{SpO}_{2}\right)$ to normal or near normal levels. Oxygen is a common part of medical care for critically sick patients. As emergency medicine care grows in Ethiopia, nursing practice has expanded to include, providing therapies such as oxygen. Our study aimed to assess understanding, belief and usage of $\mathrm{O}_{2}$ among nurses working in the emergency departments (EDs) of four public hospitals in Addis Ababa, Ethiopia.

Methods: A structured self-administered questionnaire was used to assess knowledge, attitudes, and practices. The collected data was analyzed using SPSS version 20.0.Frequency distributions, cross-tabulations and a graph were used to describe the results of the study.

Results: A total of 152 nurses completed the survey. The mean score of knowledge, attitude and practice (KAP) with regards to $\mathrm{O}_{2}$ use was $3.03(\mathrm{SD}=1.6665), 13.76(\mathrm{SD}=2.102)$ and $3.0921(\mathrm{SD}=1.76183)$ respectively; $38.2 \%$ of participants were males and their median age was $26-30$ years. The level of KAP of oxygen therapy was poor and good in $97(63.8 \%)$ and $55(36.2 \%)$ of nurses with regards to knowledge; 71 (46.7\%) and $81(53.3 \%)$ with regards to attitude; and $86(56.6 \%)$ and 66 (43.4\%) with regards to practice.

Conclusion: This study showed that there are knowledge, attitude, and practice gaps among nurses who were working in an ED of a public hospital in Addis Ababa. The possible associated factors for this gap were also identified, which included lack of proper oxygen therapy training, guidelines, workload, and inadequate supplies of oxygen delivery devices. This indicates that there is a need for increased training in this area; regular supportive supervision, health facility guideline development, and adequate oxygen devices are also needed.

Prehosp Disaster Med 2017;32(Suppl. 1):s149

doi:10.1017/S1049023X17004113

Swedish Prehospital Emergency Nurses Prepardness to Care for Pediatric Trauma Patients

Lina Gyllencreutz ${ }^{1}$, Karin Bölenius ${ }^{1}$, Britt-Inger Saveman ${ }^{2}$

1. Nursing, Umeå university, Umeå/Sweden

2. Department Of Surgical And Perioperative Sciences, Section Of Surgery; Department Of Nursing, Umeå University, Umeå/Sweden

Study/Objective: To explore prehospital emergency nurses' preparedness care for pediatric trauma patients. 